University of New Hampshire

University of New Hampshire Scholars' Repository

Space Science Center

Institute for the Study of Earth, Oceans, and

Space (EOS)

10-19-1999

\title{
Energy and position resolution of a CdZnTe gamma-ray detector with orthogonal coplanar anodes
}

O Tousignant

University of Montreal

L A. Hamel

University of Montreal

Pierre C. Dufour

University of Montreal

F Joly

University of Montreal

John R. Macri

University of New Hampshire - Main Campus, John.Macri@unh.edu

See next page for additional authors

Follow this and additional works at: https://scholars.unh.edu/ssc

Part of the Astrophysics and Astronomy Commons

\section{Recommended Citation}

Olivier Tousignant ; Louis-Andre Hamel ; Pierre C. Dufour ; F. Joly ; John R. Macri ; Mark L. McConnell ; James M. Ryan and Valentin T. Jordanov "Energy and position resolution of a CdZnTe gamma-ray detector with orthogonal coplanar anodes", Proc. SPIE 3768, Hard X-Ray, Gamma-Ray, and Neutron Detector Physics, 38 (October 19, 1999); doi:10.1117/12.366613; http://dx.doi.org/10.1117/12.366613

This Conference Proceeding is brought to you for free and open access by the Institute for the Study of Earth, Oceans, and Space (EOS) at University of New Hampshire Scholars' Repository. It has been accepted for inclusion in Space Science Center by an authorized administrator of University of New Hampshire Scholars' Repository. For more information, please contact Scholarly.Communication@unh.edu. 


\section{Authors}

O Tousignant, L A. Hamel, Pierre C. Dufour, F Joly, John R. Macri, Mark L. McConnell, James M. Ryan, and V T. Jordanov 


\title{
Energy and position resolution of a CdZnTe $\gamma$-ray detector with orthogonal coplanar anodes
}

\author{
O. Tousignant ${ }^{a}$, L.A. Hamel ${ }^{a}$, P. Dufour ${ }^{a}$, F. Joly ${ }^{a}$, J.R. Macri ${ }^{b}$ \\ M.L. McConnell ${ }^{b}$, J.M. Ryan $^{b}$, V.T. Jordanov ${ }^{c}$ \\ ${ }^{a}$ GCM, Physics Department, University of Montreal, Montreal, Canada \\ ${ }^{b}$ Space Science Center, University of New Hampshire, Durham, NH, USA \\ ${ }^{c}$ Yantra, 12 Cutts Rd., Durham, NH, USA
}

\begin{abstract}
We report on the simulation, construction and performance of prototype CZT imaging detectors employing orthogonal coplanar anodes. These detectors employ a novel electrode geometry with non-collecting anode strips in one dimension and collecting anode pixels, interconnected in rows, in the orthogonal dimension. These detectors retain the spectroscopic and detection efficiency advantages of single carrier (electrons) charge sensing devices as well as the principal advantage of conventional strip detectors with orthogonal anode and cathode strips, i.e. an $N \times N$ array of imaging pixels are realized with only $2 N$ electronic channels. Charge signals induced on the various electrodes of a prototype detector with $8 \times 8$ unit cells (of $1 \times 1 \times 5 \mathrm{~mm}^{3}$ ) are in good agreement with the simulations. The position resolution is about $1 \mathrm{~mm}$ in the direction perpendicular to the pixel lines while it is of the order of $100 \mu \mathrm{m}$ in the other direction. Energy resolutions of $0.9 \%$ at $662 \mathrm{keV}, 2.6 \%$ at $122 \mathrm{keV}$ and $5.7 \%$ at $60 \mathrm{keV}$ have been obtained at room temperature.
\end{abstract}

Keywords: CdZnTe, detector, strip detector, charge transport, signal generation, simulation, imaging, gamma-ray, spectroscopy

\section{INTRODUCTION}

\subsection{Motivation}

Imaging spectrometers utilizing cadmium zinc telluride (CdZnTe or CZT) detectors are under study at UNH for two astrophysical applications, the Minute of Arc Resolution Gamma-Ray Imaging Experiment (MARGIE) and the Tracking and Imaging Gamma Ray Experiment (TIGRE). MARGIE represents an effort to provide a large field of view survey instrument with a 2-6 arc-minute angular resolution for the $30-300 \mathrm{keV}$ energy range. ${ }^{1}$ For that experiment, closely-packed arrays of imaging CZT modules are under consideration for use in the central detector plane of a coded aperture instrument. The Tracking and Imaging Gamma Ray Experiment, or TIGRE, will use several (5-10) layers of cadmium zinc telluride (CZT) strip detectors as the second component of a Compton telescope for measurements in the $0.3-10 \mathrm{MeV}$ range. ${ }^{2}$ For both these applications, we are pursuing modular CZT detector designs optimised for good detection efficiency, energy resolution and position resolution performance.

Good efficiency, energy resolution and position resolution have been demonstrated in pixellated CZT detectors up to $10 \mathrm{~mm}$ thick. ${ }^{3}$ These detectors are "electron-only" devices, thus avoiding the deleterious consequences of poor hole transport in CZT. They employ an anode pixel contact geometry and require an electronic channel for each pixel. Strip detectors, on the other hand, offer the important advantage of providing $N \times N$ pixels with only $2 N$ electronic channels. In our earlier work with CZT strip detectors employing orthogonal anode and cathode strips we reported an important detection efficiency limitation related to the poor transport of holes in CZT material. ${ }^{4}$ Photons interacting far from the incident cathode surface produce small cathode strip signals. These signals are required to identify the interaction location in one dimension. This limitation restricts the thickness of the CZT and is particularly significant when the energy range extends above a few hundred $\mathrm{keV}$. Orthogonal anode and cathode

Further author information:

O.T.: Email: tousi@lps.umontreal.ca; Telephone: (514) 343-6204; Fax: (514) 343-6215

J.R.M.: Email: John.Macri@unh.edu; Telephone: (603) 862-2793; Fax: (603) 862-4685. 

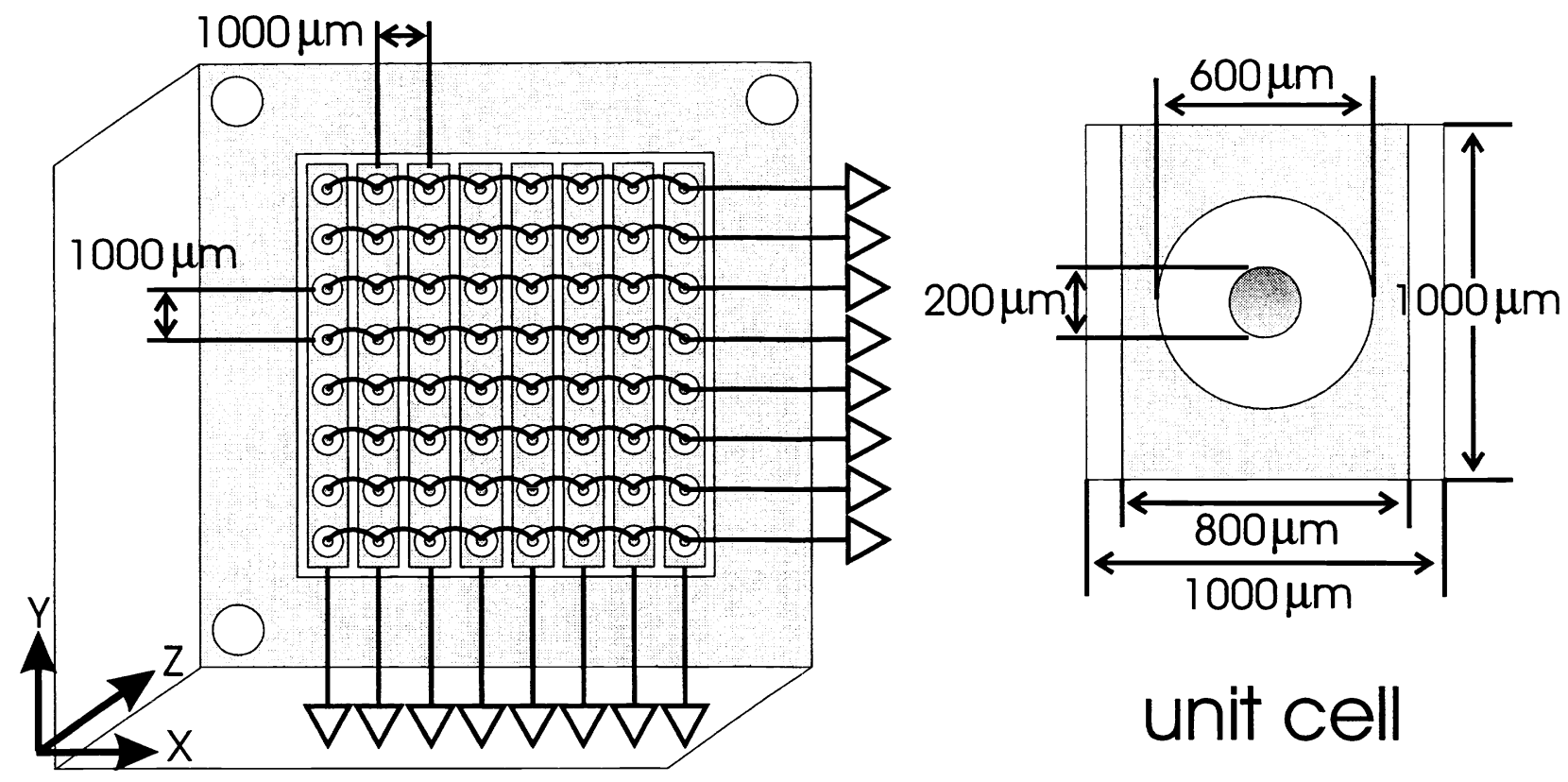

Figure 1. Schematic diagram showing the layout of the orthogonal coplanar anode design along with an enlarged view of a unit cell. The dimensions shown here correspond to those of the prototype assemblies.

strip detectors, if carefully designed, can address these limitations. ${ }^{5}$ There will, however, be an upper limit on detector thickness and thus on the effective energy range. Orthogonal anode and cathode strip detectors also have the requirement that electrical contact be made to the strips on both the incident (cathode) and underside (anode) surfaces of the CZT substrate. This adds to dead space in assembling closely packed arrays of imaging modules.

\subsection{Detector Design}

To address these problems as well as to explore new technologies for fabricating imaging CZT detectors, we have been investigating and developing a CZT detector concept, an "electron-only" device featuring orthogonal coplanar anodes. ${ }^{6-8}$ The rows take the form of discrete inter-connected anode pixels while the columns are non-collecting strips in which the pixels are embedded. Figure 1 illustrates the anode surface contact pattern and the readout lines of an $8 \times 8$ orthogonal coplanar anode detector. The figure also defines the axis system used in this paper. The opposite side, not shown, has one uniform cathode electrode. The anode pixels, interconnected in rows, are biased to collect the electrons. The orthogonal strips surrounding the anode pixels are biased at a level intermediary between the cathode and pixel potentials to focus the incoming electrons toward the pixel. They also measure induced signals from electrons drifting to the anode plane. The energy and position information in both directions are thus obtained exclusively from the anode signals (pixels and strips) and photon interactions at all depths in the detector will be detected. A simulation of this device ${ }^{7}$ indicated that good energy resolution was achievable, that the strip signals were large enough to provide the position information in the $X$-direction and that some position information in the $Z$-direction could also be extracted. This will permit thicker, more efficient CZT imaging planes than are practical with double sided strip detectors having orthogonal anode and cathode strips. This will extend the effective energy range to $>500 \mathrm{keV}$. In addition, more compact packaging is possible since all imaging contacts and connections are on the same side of the detector.

\subsection{Present Work}

In this paper, we report on preliminary results obtained with the first prototype of the orthogonal coplanar anode detector. The position resolutions in 2 dimensions have been measured by scanning a collimated $\alpha$ source over a small area of the cathode. The energy resolution for $\gamma$-rays has been measured in a $1 \times 1 \mathrm{~mm}^{2} \mathrm{unit}^{\text {cell }}$ with $^{241} \mathrm{Am}$, ${ }^{57} \mathrm{Co}$ and ${ }^{137} \mathrm{Cs}$ sources. Signals on the pixels and strips produced by $\alpha$-rays and $\gamma$-rays have been measured and compared with simulations. 


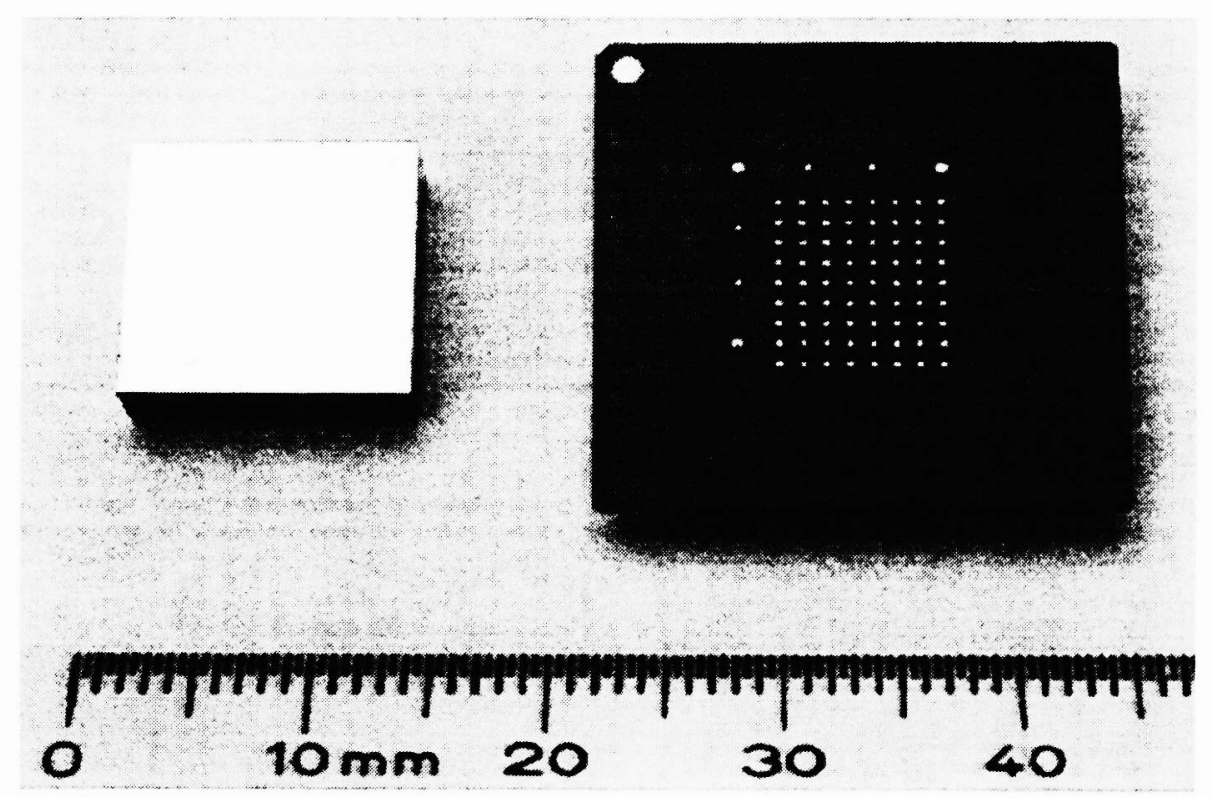

Figure 2. Detector prototype components. Photograph of a patterned CZT substrate (left) and its mating ceramic carrier.

\section{DETECTOR AND MEASUREMENT SETUP}

\subsection{Detector Fabrication}

The first two prototypes employing the orthogonal coplanar anode contact geometry have been fabricated. Figure 2 is a photograph of the prototype components: a patterned CZT substrate (left) and its mating ceramic carrier. Assembly involves electrical and mechanical bonding between the two surfaces shown in the photograph. The CZT substrate is $12 \mathrm{~mm}^{2}$ and $5 \mathrm{~mm}$ thick. Anode pixel contact pads are $200 \mu \mathrm{m}$ in diameter and the pitch is $1 \mathrm{~mm}$ in each dimension. The prototypes have 8 pixel rows $\times 8$ strips forming 64 unit cells. The active area is surrounded by a large guard ring.

A low temperature bonding process well suited to the special handling requirements for CZT is under study and has been used for the first time to form the electrical and mechanical connection of the patterned CZT and ceramic substrates of our prototype detector assemblies. The result is a rugged assembly that involves no wire bonds to the CZT anode surface. A paper providing the details of this process is in preparation.

The first two prototype assemblies were completed very recently (April, 1999). Figure 3 shows one of these. They were fabricated using inexpensive, multi-crystal, counter grade CZT material from eV Products. This material was used to help minimise the initial risks associated with exercising and evaluating the bonding process with CZT. More expensive single crystal and spectroscopy grade patterned CZT substrates procured from eV Products and Digirad are also available and will be used in subsequent studies.

\subsection{Electronics}

A front end electronics circuit and test box was fabricated to conduct the tests and measurements reported here. The circuit board employs sockets so that available detector assemblies can be inserted and removed. The box cover can be removed to allow measurements with a collimated beam of alpha particles incident on the cathode surface. The circuit permits separately adjustable bias settings for the cathode, the anode strips and the guard ring. The pixels are held at zero potential. The cathode, strips and pixel rows are AC-coupled to $\mathrm{eV}-5093$ charge sensitive preamplifiers with a sensitivity of $3.6 \mathrm{mV} / \mathrm{fC}$. No additional analog signal processing is performed on the preamplifier outputs. A common test input allows the injection of charge from a pulser on all preamplifier channels simultaneously.

\section{MEASUREMENTS AND RESULTS}

The results reported here are from measurements made on one of the prototypes described in the previous section. Various types of measurements were performed with $\alpha$ and $\gamma$ sources. Collimated $\alpha$-rays from a ${ }^{244} \mathrm{Cm}$ source were used to scan the detector across the cathode to study the position resolution for interactions occurring at the cathode $(Z=0)$. Signals produced by the $\alpha$-rays were also used to measure the mobilities and lifetimes of electrons and 


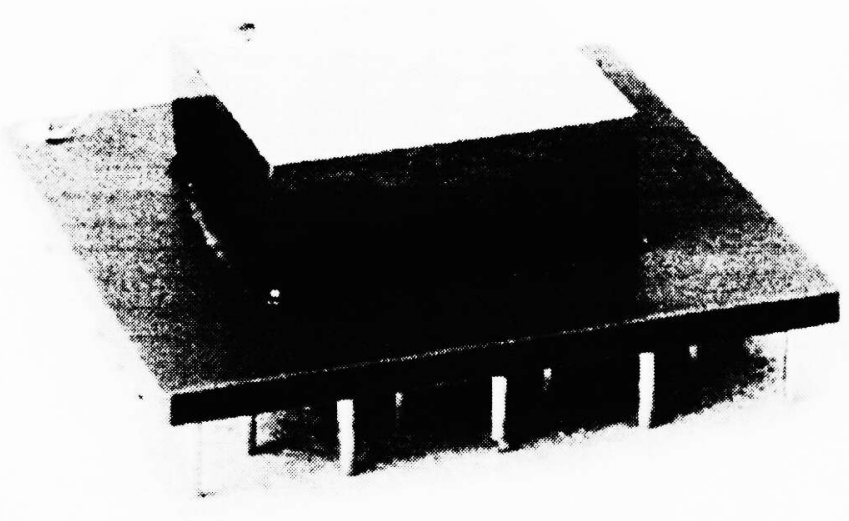

Figure 3. First prototype detector assembly. A $5 \mathrm{~mm}$ thick patterned CZT substrate is bonded to its mating ceramic substrate. Signals are broken out to pins on a PGA pattern for testing with laboratory electronics.

holes. $\gamma$-rays from various sources $\left({ }^{241} \mathrm{Am},{ }^{57} \mathrm{Co},{ }^{137} \mathrm{Cs}\right)$ were used to measure the energy resolution and to study the response to interactions in the detector volume.

Two types of measurement setups were used. First, for pulse height measurements, the preamplifier signals were fed to standard spectroscopy amplifiers and a multichannel analyser (MCA). For measurements in which the shape of the charge signals on the various electrodes had to be analysed, the preamplifier signals were sent directly to two digital sampling oscilloscopes (Tektronix TDS360). The scope traces were then readout and processed on a PC.

For both setups, a trigger electronics selected events occurring in a given unit cell. To that end, a coincidence was made between a pixel line and a strip to generate a gate that was sent to the MCA or to the external trigger of both scopes.

\subsection{Preliminary Measurements}

First measurements with ${ }^{57} \mathrm{Co}$ and ${ }^{137} \mathrm{Cs}$ sources indicated that some pixel lines and strips had better responses than others as one expects from a multi-crystal detector. Three adjacent pixel rows (labelled P3 to P5) and three strips (S3 to S5) gave good signals. The region defined by the intersection of these channels corresponds to a $3 \times 3 \mathrm{~mm}^{2}$ area in the central region of the detector. The unit cell P4-S4 was therefore selected for most subsequent measurements.

The operating bias voltage on the various electrodes were determined by optimising the energy resolution. With the pixels held at zero Volts, the (negative) voltages on the cathode and strips were raised to maximise the pixel signal until a full collection plateau for the pixel was reached but before the leakage current (especially between strip and pixel) deteriorated the resolution. A cathode bias of -800 Volts and a strip bias of -30 to -70 Volts gave best results. The guard ring was biased at the strip potential.

\subsection{Collimated $\alpha$-ray Scans}

A collimated ${ }^{244} \mathrm{Cm}$ source was mounted on a 3-axis micro-manipulator and positioned $0.4 \mathrm{~mm}$ from the cathode. The illuminated spot on the detector face was about $200 \mu \mathrm{m}$ in diameter. The source was scanned horizontally across the strips ( $X$-coordinate) or vertically across the pixel rows ( $Y$-coordinate) while the signals from pixel and strip channels were recorded on the scopes. For each event, the total charge collected on the pixel channels is determined using a digital triangular ${ }^{\ominus}$ shaping with a measurement time of $500 \mathrm{~ns}$. The peak of the strip signals was also determined from the traces. The values were stored in a file for further analysis.

A preliminary horizontal scan was made to find the center of strip S4 where the S4 signal is maximum and the neighbouring S3 and S5 signals are equal. The detector was then scanned vertically in the center of S4 in steps of $100 \mu \mathrm{m}$. Signals on P3, P4, P5 and S4 were measured. At each point, 500 events were recorded. Fig. 4 shows how the mean amplitudes (normalised at each event) vary with source position. 

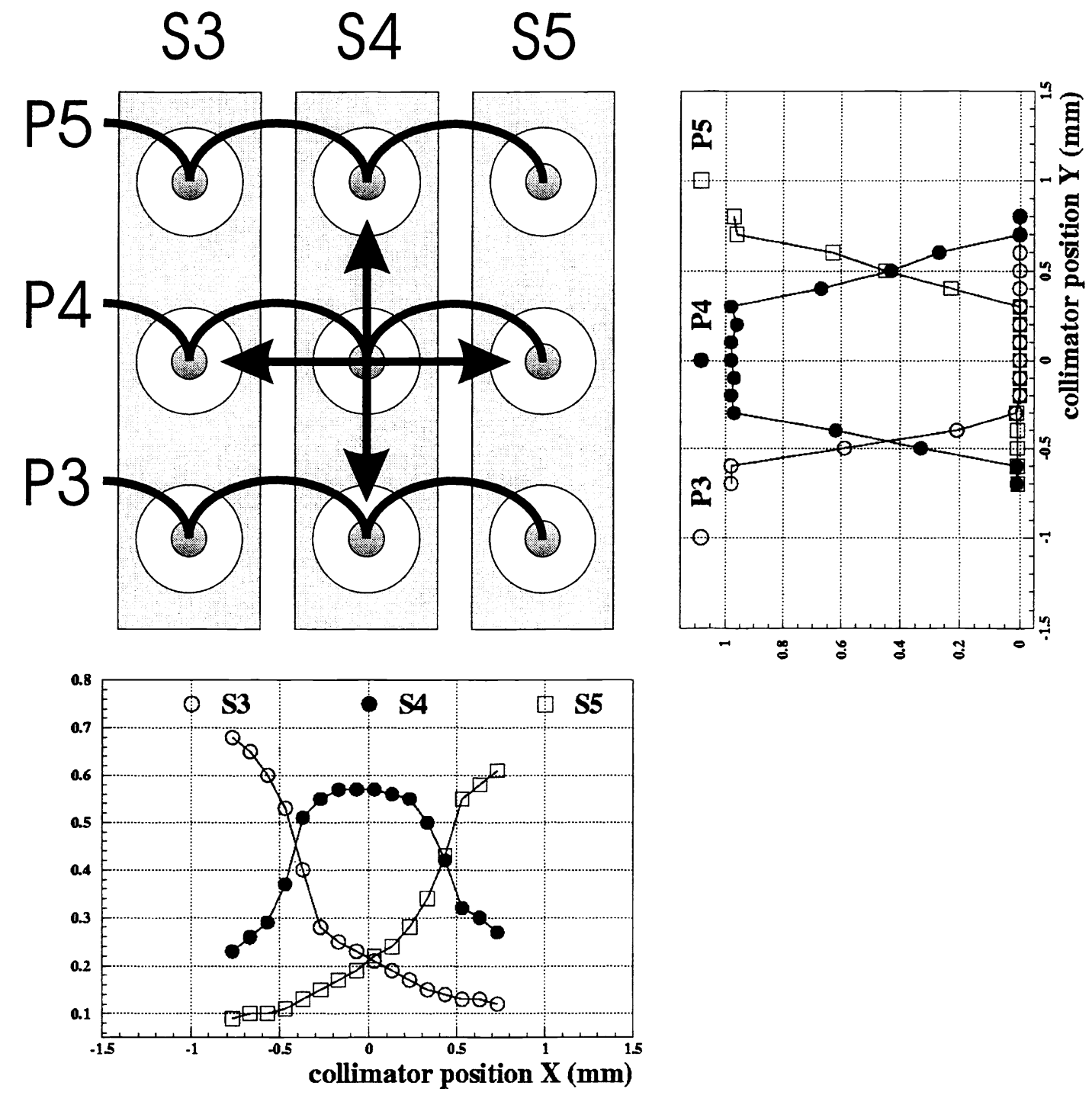

Figure 4. Collimated $\alpha$-ray scans. The scan lines in the horizontal and vertical directions are depicted by the arrows in the sketch of a small region of the detector (top, left). For both scans, the relative amplitudes on three adjacent channels (normalised to their sum) are plotted as a function of the source position. The $Y$-scan plot (top, right) has been rotated to align the $Y$-axis with the scan direction in the detector sketch.

A similar scan in the horizontal direction was made in the center of P4 while P4, S3, S4 and S5 signals were recorded. Figure 4 shows the result of this scan. Some charge sharing is observed for scans in the vertical and horizontal directions. In the horizontal direction, the amplitude on the 3 strips vary slowly and continuously over the whole scan. But in the vertical direction, pixels are almost insensitive to events occurring in the adjacent rows and the pixel amplitudes are constant (either 0 or $100 \%$ ) inside a unit cell, the sharing being restricted to a small region between the pixel lines. As a consequence, there is little sub-cell $Y$-position information and the vertical resolution is of the order of $1 \mathrm{~mm}$. On the other hand, a much better position information can be inferred in the $X$-direction. This is due to the fact that a strip, because it covers a larger area than a pixel line, is sensitive to electrons moving at a greater distance. The smooth decrease of S3 and increase of S5 when the source is moved to the right, inside the region covered by S4, makes the difference S5-S3, normalised by the sum S3+S4+S5, a good position evaluator.

Fig. 5 shows that the quantity (S5-S3)/(S3+S4+S5) varies linearly with $X$ across S4 and tends to deviate from a straight line only in the gaps. It is also observed that (S5-S3)/(S3+S4+S5) is independent of $Y$ since results from the preliminary horizontal scan, made $300 \mu \mathrm{m}$ above the P4 line, fall on the same line. The straight line in fig. 5 


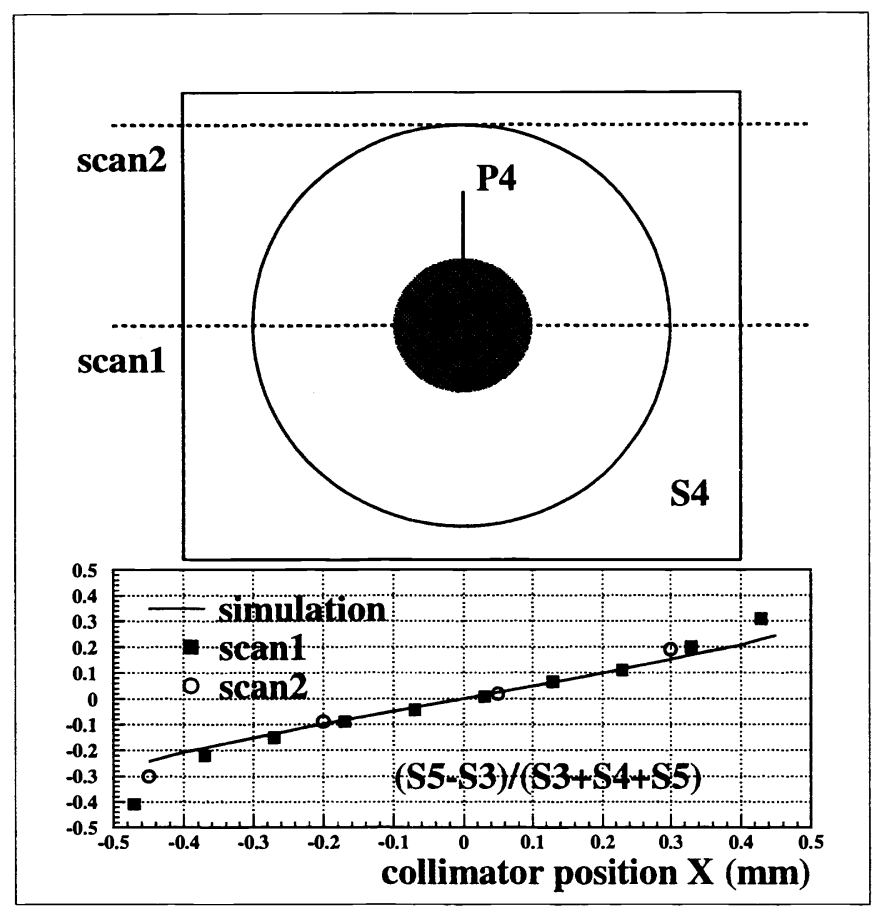

Figure 5. Sub-cell position resolution in the $X$-dimension. The quantity (S5-S3)/(S3+S4+S5) formed from the signal amplitudes on the strips $\mathrm{S} 3$ to $\mathrm{S} 5$ is plotted as a function of $X$. The results are shown for 2 scans at different $Y$-positions. The straight line is the prediction from the simulations.

represents the results obtained by analysing the simulated signals in the same way (the simulations will be presented in section 3.4). The position resolution in $X$ is thus much finer than the pitch. The standard deviation of the (S5-S3)/(S3+S4+S5) distribution at a given point is about $100 \mu \mathrm{m}$. This dispersion is mostly due to the spot size and the actual position resolution is probably better than $100 \mu \mathrm{m}$.

\section{3. $\gamma$-ray Spectra}

Energy spectra measured in the unit cell S4-P4 for three $\gamma$-ray sources are shown in fig. 6. In two cases, a pulser peak has also been collected to evaluate the electronic noise. The FWHM resolution at $662 \mathrm{keV}$ is $0.9 \%$ or $5.9 \mathrm{keV}$. The pulser FWHM is $3.3 \mathrm{keV}$. A small X-ray escape peak is visible around channel 305 . The resolution is $2.6 \%$ or $3.2 \mathrm{keV}$ at $122 \mathrm{keV}$ and $2.2 \%$ or $3 \mathrm{keV}$ at $136 \mathrm{keV}$. In this case, the pulser FWHM was $2.8 \mathrm{keV}$. A very good separation of the 122 and $136 \mathrm{keV}$ is observed. The resolution is $5.7 \%$ or $3.4 \mathrm{keV}$ at $60 \mathrm{keV}$. The energy resolution in the present device, at low energy, is presently limited by the electronic noise and could possibly be improved. If the noise is quadratically subtracted from the resolution, an intrinsic resolution of $1.5 \mathrm{keV}$ at $122 \mathrm{keV}$ has been achieved which must be compared to the theoretical limit, from statistics only, of $2.35 \sqrt{F \epsilon E}=0.6 \mathrm{keV}$ if values of $F=0.1$ and $\epsilon=5 \mathrm{eV}$ are used for the Fano factor and the pair creation energy in CZT.

The continuum between the ${ }^{137} \mathrm{Cs}$ photopeak and its Compton edge is due to events in which only a fraction of the electrons were collected on P4. As seen from the vertical $\alpha$-ray scan (fig. 4 (top, right)), there is a region between two pixel lines in which the charge is shared between the two channels. The linear decrease of P4 with $X$ is expected to produce a uniform low energy tail to the photopeak. Partial charge collection on the strips for some events also contributes to the low energy continuum. Similar low energy tails are also visible for the 60,122 and 136 $\mathrm{keV}$ peaks. By summing the amplitudes from the two adjacent pixels for these events, most of the continuum should be removed and brought back into the full energy peaks. 

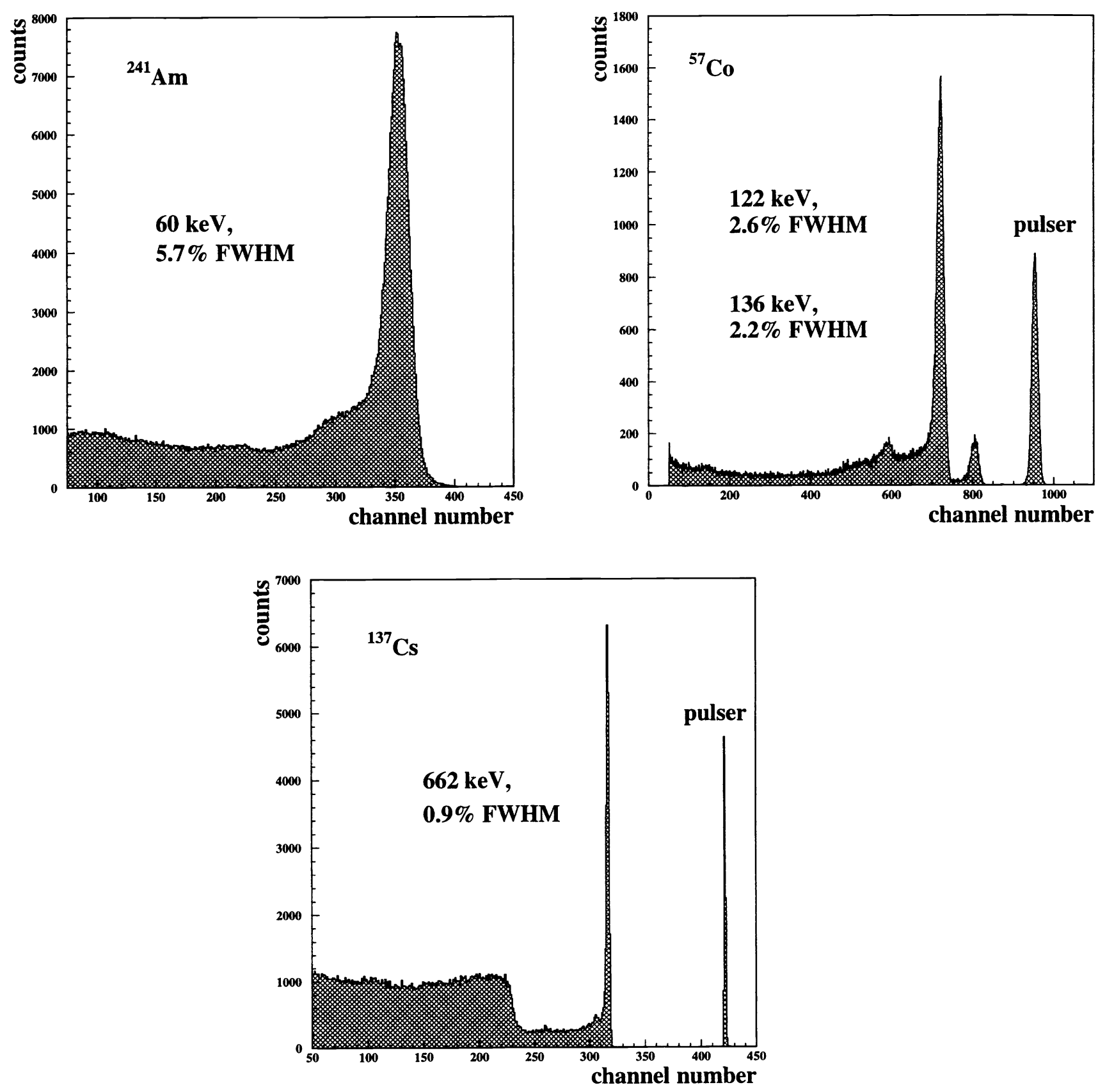

Figure 6. Energy spectra for ${ }^{241} \mathrm{Am},{ }^{57} \mathrm{Co}$ and ${ }^{137} \mathrm{Cs} \gamma$-ray sources.

\subsection{Comparison of Measured and Calculated Signals}

Induced charge signals measured on the scopes for $\alpha$ and $\gamma$-rays are compared with simulations. The simulation procedure for this device is detailed in ref. ${ }^{7}$ The electron transport parameters used are a mobility $\mu_{e}=1200$ $\mathrm{cm}^{2} \mathrm{~V}^{-1} \mathrm{~s}^{-1}$, a deep trapping lifetime $\tau=4 \mu \mathrm{s}$, a shallow trapping time $\tau_{t}=40 \mathrm{~ns}$ with a detrapping time of $\tau_{d}=10$ ns. Shallow trapping and detrapping times short compared to the transit time simulate carrier dispersion and reduce the mobility to an effective value of $960 \mathrm{~cm}^{2} \mathrm{~V}^{-1} \mathrm{~s}^{-1}$. 


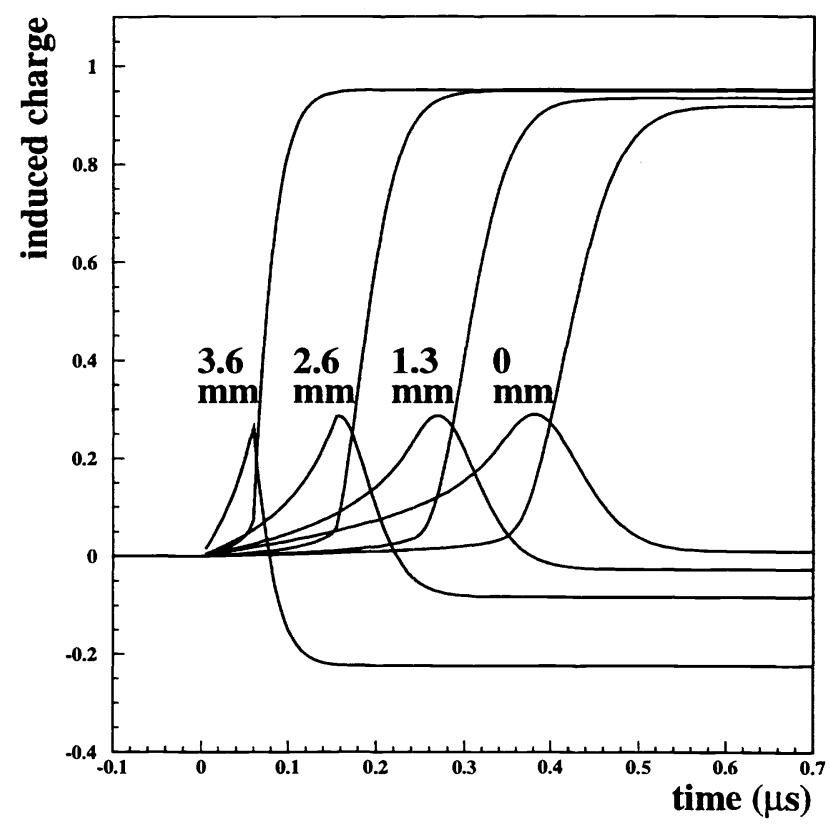

Figure 7. Calculated pixel and strip signals for interactions at various depths (measured from the cathode) along the central axis of a unit cell. The signals are normalized to the deposited energy.

Fig. 7 presents simulated strip and pixel signals for interactions at 4 different depths along the central axis of a unit cell. In each case, the initial slope of the pixel signal is small. The signal increases very rapidly when electrons reach the anode region. The pixel risetime is a short fraction of the total transit time and increases slightly for longer transit times due to carrier dispersion. The maximum amplitude reached on the pixel depends on the position of interaction $\vec{x}_{0}$ because of electron and hole trapping. Signal deficit due to electron trapping increases with transit time and, in the present simulations, is responsible for the $\sim 7 \%$ signal deficit at $Z=0$, the loss being less important at other locations. Since holes are trapped almost immediately at $\vec{x}_{0}$, they contribute little induced current and are responsible for an induced charge deficit on electrode $i$ that is proportional to $V_{W i}\left(\vec{x}_{0}\right)$, the weighting potential of that electrode at $\vec{x}_{0}$. Signal deficit from hole trapping is zero at $Z=0$ and increases with $Z$. The two effects have opposite dependences on $Z$ and partially compensate each other.

Strip signals have faster initial rises than pixel signals due to the strip larger areas. Strip signals reach a transient maximum shortly before transit time and decrease when the electrons approach the pixel. That maximum signal sensed by the strip is nearly independent of $Z$ for interactions on the central axis (fig. 7). The negative residual charge left on the strip at the end of the event is due to trapped holes and is more and more negative at larger $Z$.

Simulated signals will now be compared to traces recorded on the scopes. First, signals P4, S3, S4 and S5 for an $\alpha$-ray incident in the center of the unit cell P4-S4 $(X=0, Y=0)$ were recorded and compared with the simulation (fig. 8a). Another similar comparison was made at $X=450 \mu \mathrm{m}$ and $Y=0$ (fig. 8b). In each case an overall normalisation factor and time translation, adjusted to the pixel signal, were applied to the 4 experimental signals. The apparent larger noise at $X=450 \mu \mathrm{m}$ is due to a smaller collected signal in that particular event that has been normalised to the same level. The shapes and relative sizes of the 4 signals are well predicted by the simulations for both locations. The most notable difference between these events is the relative reduction of S3 and increase of S5 when the source is moved from $X=0$ to $X=450 \mu \mathrm{m}$. 

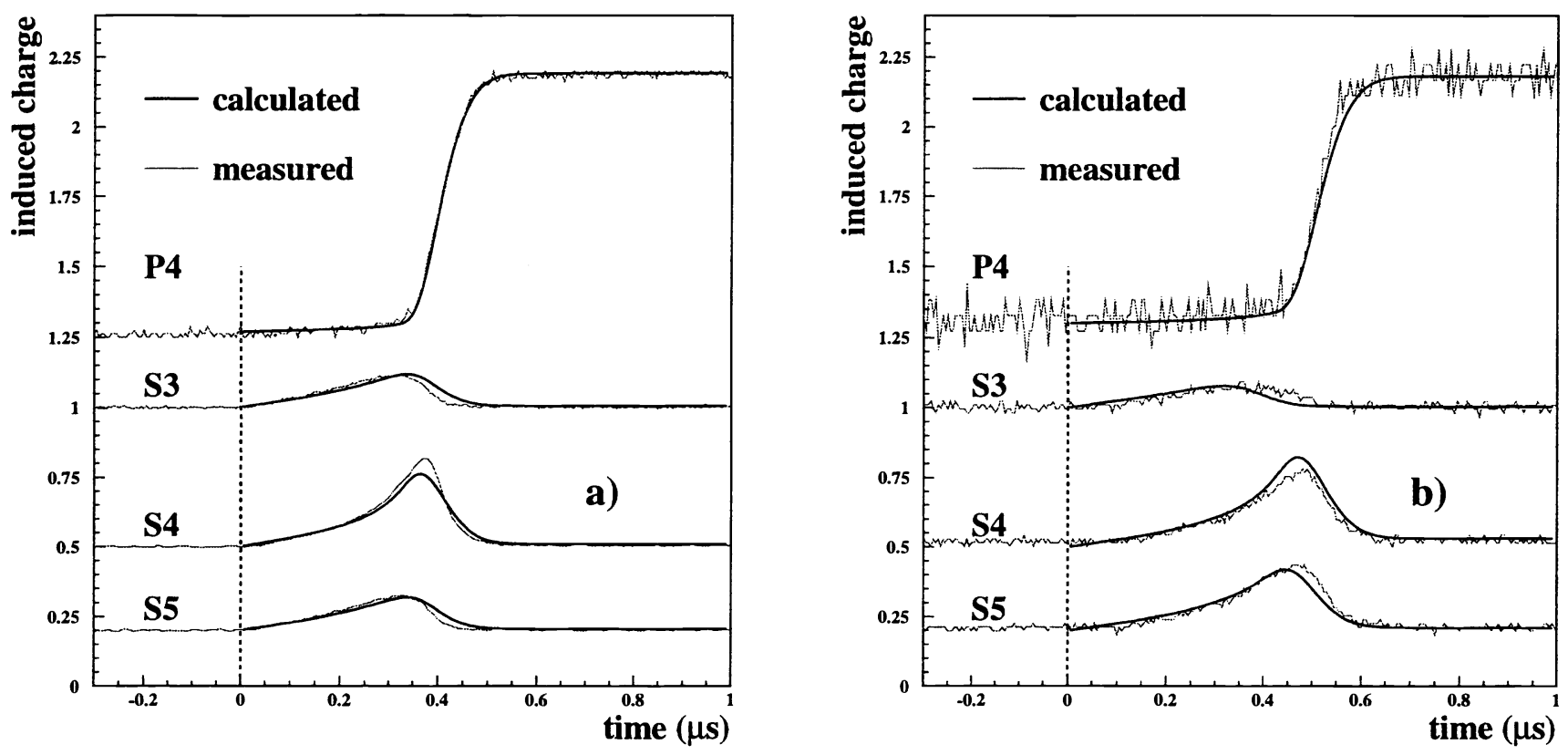

Figure 8. $\alpha$-ray events occurring in the unit cell P4-S4 : a) in the center of the cell and b) $450 \mu \mathrm{m}$ to the right.

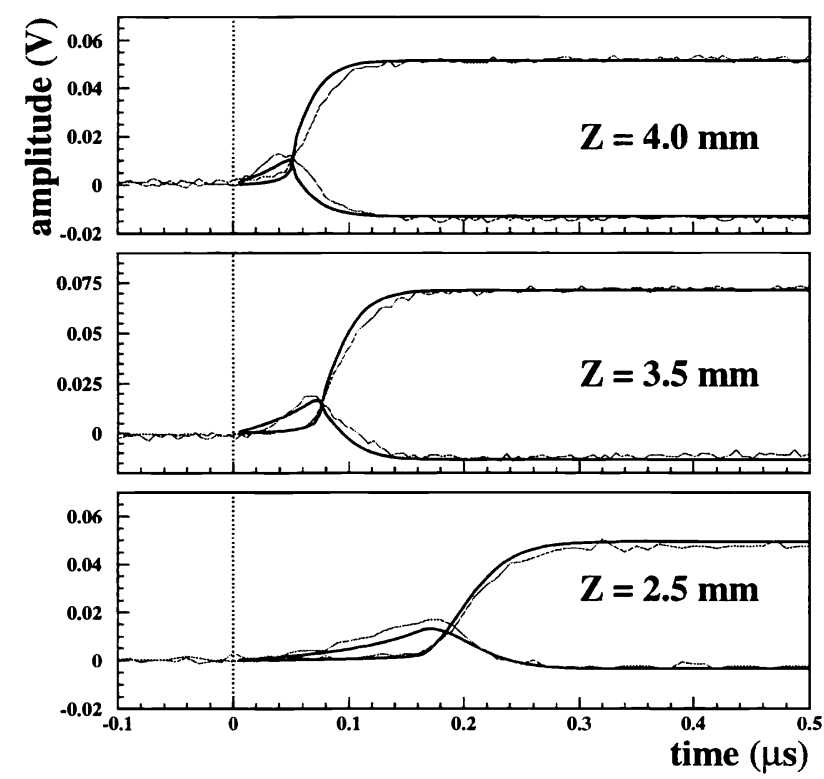

Figure 9. Measured and simulated signals from ${ }^{137} \mathrm{Cs} \gamma$-rays interacting at three different depths. The $Z$ positions have been inferred from the simulations.

In order to compare experimental traces to the simulations shown in fig. 7, at different depths, traces on P4, S3, S4 and S5 were recorded with a ${ }^{137}$ Cs source. Three events were selected for which S3 and S5 were identical, ensuring that they occurred in the center of S4, and with different transit times and strip signal shapes, indicating different locations in $Z$. The approximate value of $Z$ was determined by comparison with simulated signals. The 
result of that comparison is shown in fig. 9. Signal shapes, relative amplitudes and transit time are well reproduced by the simulations.

\section{DISCUSSION}

First measurements with this prototype detector have already demonstrated that the orthogonal coplanar anode design is promising. The expected performance in energy and position resolution has been met or exceeded. Resolutions better than $1 \%$ at $662 \mathrm{keV}$ and $3 \%$ at $122 \mathrm{keV}$ have been achieved from the pixel signals only with no trapping correction from the strip or cathode information. The energy resolution is presently limited by the electronic noise and will possibly be improved. This excellent intrinsic resolution is the result of partial compensation, in this electrode geometry, between the effects of electron and hole trapping.

The position resolution in the $Y$-dimension is of the order of the pitch between the pixel lines and a much finer resolution has been observed in the other dimension. Some depth sensitivity has also been demonstrated.

The experimental signals are well reproduced by the simulations. This agreement is a good test of the simulation procedure that can now be used confidently for future design optimisation and for further analysis of the experimental data. The energy and position resolutions exceed the design goals of both the MARGIE and TIGRE applications. ${ }^{1,2}$

Given the success with the first prototypes made from low grade material, we will now fabricate and test several detector assemblies employing single crystal CZT material. Detectors with $10 \mathrm{~mm}$ thick CZT substrates will also be fabricated and tested. The supporting electronics will include a bias compensation circuit ${ }^{6}$ on each strip to compensate for differences in leakage current and establish more uniform fields. The data acquisition system for the laboratory studies will be expanded to permit the recording, digital filtering and analysis of all pixel row and orthogonal strip signals for each event.

Measurements of efficiency, energy and position resolution will be performed over the whole detector. These will include measurements of the capability to determine the event location in the third spatial dimension, the interaction depth, and to reduce the low energy continuum in the $\gamma$-ray spectra by summing adjacent pixel signals for interactions close to cell boundaries. One of these protoypes will be used as a second detector in a Compton spectrometer for evaluation of a complete imaging system. These laboratory studies, together with charge transport and signal generation simulations, will provide an understanding of the nature of the detector signals which will in turn facilitate development of complete systems (detector and electronics) required for flight instruments.

\section{CONCLUSIONS}

A prototype of a CZT imaging detector with orthogonal coplanar anodes has been fabricated. First measurements confirmed that this new detector could provide excellent energy resolution and imaging performance. Signals observed on the pixels and strips are in good agreement with the simulations.

More prototypes will be fabricated with better CZT material and a dedicated data acquisition system will be developed to record all 16 channels for further studies. Future measurements will first concentrate on studying the detector response but will also include tests of more complete systems in several imaging applications.

\section{ACKNOWLEDGEMENTS}

We thank Dr. Ralph James of Sandia National Laboratories for his help with procurement of the CZT substrates. This work is supported by NASA's High Energy Astrophysics Gamma Ray Astronomy Research and Analysis program and by the Natural Sciences and Engineering Research Council (NSERC) of Canada.

\section{REFERENCES}

1. M. L. Cherry, et al., "A New Balloon-Borne Detector for High Angular Resolution Hard X-Ray Astronomy", in Proceedings, 24th International Cosmic Ray Conference, Rome, August 1995.

2. Zych, A. D., D. Bhattacharya, D. Dixon, T. O'Neill, T. Tumer, R. S. White, J. Macri, M.McConnell, J. Ryan, H. Ogelman, R. Paulos, W. Wheaton, A. Akyuz, J. Samimi and M. Ozel, "Few arcminute and keV resolutions with the TIGRE Compton Telescope", Proc. of the 2nd INTEGRAL Workshop , European Space Agency (ESA) SP-382, March, pp. 367-370, 1997. 
3. He, Z. et al., "3-D position sensitive CdZnTe gamma-ray spectrometers", Nucl. Instr. and Meth. A422, pp. 173-178, 1999.

4. Mayer, M., et al., " Performance and Simulation of CdZnTe Strip Detectors as Sub-millimeter Resolution Imaging Gamma Radiation Spectrometers", IEEE Trans. Nucl. Sci., 44, pp. 922-928, 1997.

5. Matteson, J.L., et al., Proc. SPIE 3446, 192, 1998.

6. Jordanov V.T., et al., "Biasing Scheme for AC Coupled Strip Detectors", IEEE Trans. Nucl. Sci.,vol. 45, No. 3, June 1998.

7. Hamel, L.A., et al., "An imaging CdZnTe detector with coplanar orthogonal anode strips", Proceedings of th 1997 Fall Meeting of the Material Research Society, Boston, 1-5 December, 1997.

8. Mayer, M., et al., "Signal Formation in a CdZnTe Imaging Detector with Coplanar Pixel and Control Electrodes", Nucl. Instr. and Meth. A422, pp. 190-194, 1999.

9. Jordanov V.T., Knoll G.F., "Digital Synthesis of pulse shapes in real time for high resolution radiation spectroscopy", Nucl. Instr. and Meth. A345, pp. 337-345, 1994. 